\title{
CARACTERIZACIÓN DE CONCENTRADOS DE COBRE PRODUCIDOS EN ARGENTINA PARA ANALIZAR LA FACTIBILIDAD DE LA INSTALACIÓN DE UNA PLANTA PIROMETALÚRGICA
}

\section{CHARACTERIZATION OF THE ARGENTINE COPPER CONCENTRATES TO EVALUATE THE POSSIBILITY OF A PIROMETALLURGY INDUSTRY INSTALLATION}

\author{
Vanesa Bazán $^{1} \quad$ Pedro Sarquis $^{2} \quad$ Elena Brandaleze $^{3} \quad$ Ivana Orozco $^{2}$ \\ Recibido 21 de septiembre de 2009, aceptado 10 de noviembre de 2010 \\ Received: September 21, 2009 Accepted: November 10, 2010
}

\begin{abstract}
RESUMEN
La demanda mundial de cobre metálico se incrementó sensiblemente durante estos últimos años debido a los mercados emergentes y a China en particular. Actualmente en Argentina los proyectos mineros de explotación de cobre que se desarrollan en el norte del país concluyen su proceso con la exportación de concentrados. En nuestro país se han realizado diferentes exploraciones y en algunos casos ya están en explotación minerales de alta ley de cobre $(0,5 \%-0,8 \%)$ libres de impurezas tales como: $\mathrm{Sb}, \mathrm{As}, \mathrm{Pb}, \mathrm{Bi}$ y $\mathrm{Hg}$. Esto indicaría que hay un gran futuro de la pirometalurgia del cobre que no se ha desarrollado aquí hasta el momento. Las condiciones del mercado argentino de cobre generan una demanda insatisfecha del metal, ya que existen plantas transformadoras de cobre metálico a productos terminados provocando una nueva importación de productos intermedios de cobre. Este trabajo propone profundizar el conocimiento y ampliar el espectro de los fundamentos técnicos con el objetivo de cubrir ese espacio del mercado. Esto puede promover que los grandes proyectos cupríferos superen la instancia de exportar concentrados proporcionando esta materia prima a una industria metalúrgica nacional que produzca cobre metálico, y de este modo evitar que se importe dicho insumo y de esta manera se satisfaga el mercado interno.
\end{abstract}

Palabras clave: Potencial minero argentino, caracterización, cobre, fundición, concentrados.

\begin{abstract}
The copper demand in the world has considerably increased during the last years due to the emergent markets, particularly the Chinese market. Nowadays, the mining projects for copper exploitation that are being developed in the North of Argentina conclude with the concentrates exports. In our country different explorations have been carried out and in some cases high law of copper minerals are being exploited (0,5\%-0,8\%). These minerals are free from impurities such as: $\mathrm{Sb}, \mathrm{As}, \mathrm{Pb}, \mathrm{Bi}$ and Hg. This would lead to think that there are good prospects for the copper pyrometallurgy that have not been developed yet in our country. The conditions for the Argentinean copper market present and unfulfilled demand for this metal, since there are industrial plants which convert metallic copper into final products leading to a new copper intermediate product import. This paper proposes to deepen the knowledge and to broaden the range of technical principles with can avoid exporting concentrates and instead it can provided raw material to a national metallurgical industry in order to produce metallic copper so that the domestic market will be supplied, thus importing raw material will no longer be necessary..
\end{abstract}

Keywords: Argentine mining potential, characterization, copper, smelting, concentrate.

\footnotetext{
1 Consejo Nacional de Investigaciones Científicas y Técnicas, Argentina (CONICET)-Instituto de Investigaciones Mineras, Facultad de Ingeniería. Universidad Nacional de San Juan, Av. Libertador Gral. San Martín 1109-Oeste-5400. San Juan. Argentina. Tel: 00-54-264-4211700 int 214. E-mail: bazan@unsj.edu.ar

2 Instituto de Investigaciones Mineras, Facultad de Ingeniería. Universidad Nacional de San Juan Av. Libertador Gral. San Martín 1109-Oeste-5400. San Juan. Argentina. Tel: 00-54-264-4211700 int 216. E-mail: psarquis@unsj.edu.ar; iorozco@unsj.edu.ar

3 Universidad Tecnológica Nacional-Facultad Regional de San Nicolás- Colón 332-CP: 2900-San Nicolás-Buenos Aires-Argentina.

E-mail: ebrandaleze@frsn.utn.edu.ar
} 


\section{INTRODUCCIÓN}

Según los datos recopilados por SEGEMAR y datos complementarios de Gutiérrez [1], Peralta [2] y Preisz [3], la República Argentina alberga un excelente potencial en depósitos minerales de variada composición. Se ubica en el sexto lugar entre los países del mundo con mayores recursos mineros, cuyas reservas la potencian para poder constituirse en un fuerte abastecedor regional y poder participar activamente en mercados extrarregionales.

La superficie estimada de potencial minero en Argentina ronda los $750.000 \mathrm{~km}^{2}$, de los cuales falta explorar y explotar el $75 \%$.

De acuerdo a Edelstein [4], la producción de minerales de cobre en Argentina en el período 2001 a 2005 ha oscilado entre $177 \mathrm{Mt}$ a $204 \mathrm{Mt}$ tal como puede apreciarse en la Figura 1. Sin embargo, en el mismo período sólo $16 \mathrm{Mt}$ han sido procesadas con refino en el país, siendo exportada la mayor parte del material concentrado.

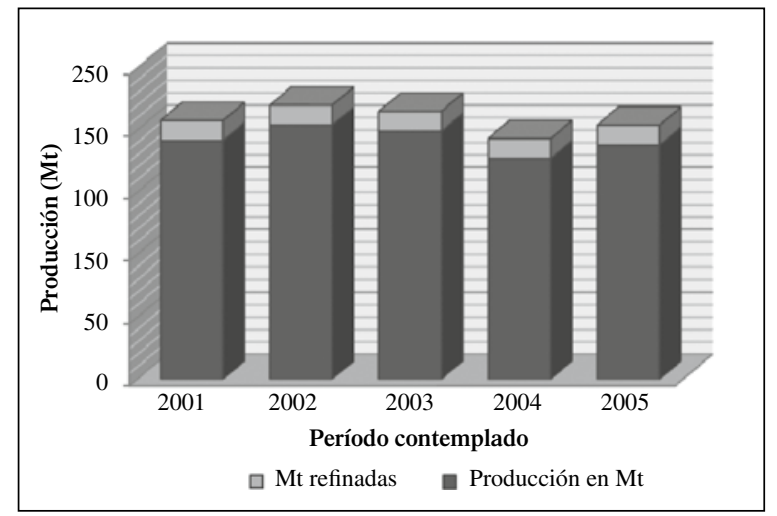

Figura 1. Producción de minera de cobre en Argentina en el período 2001 a 2005.

Las condiciones del mercado argentino de cobre generan una demanda insatisfecha del metal, ya que existen plantas transformadoras de cobre metálico a productos terminados, el cual representa un $11 \%$ del total exportado, pagando aproximadamente un $314 \%$ más del valor por el cual se vende el concentrado. Según datos del año 2009 se vende concentrado a $1,76 \mathrm{USD} / \mathrm{kg}$, mientras que se compra el cobre refinado a 5,53 USD $/ \mathrm{kg}$. Es decir, que no sólo se paga la manufactura del procesamiento de concentrado a cobre refinado, sino también la materia prima que proviene de nuestro país [5].

Si bien el precio del cobre ha disminuido en un 50\% aproximadamente con respecto al período 2007-2008, en la actualidad se observa un incremento mensual de un
8-9\%, lo cual indicaría una pronta recuperación de este metal. Sumado a este hecho, los proyectos de exploraciones y explotación de minerales de cobre que se realizan en la actualidad se caracterizan por ser de alta ley $(0,5 \%$ $0,8 \%$ ), generalmente libres de las impurezas como $\mathrm{Sb}$, $\mathrm{As}, \mathrm{Pb}, \mathrm{Bi}$ y $\mathrm{Hg}[6]$.

Lo antes mencionado permite pensar que existe un gran potencial para el desarrollo de la pirometalurgia del cobre en Argentina, a pesar de que hasta el momento no se tienen grandes avances en este sentido.

\section{DESARROLLO}

\section{Relevamiento del potencial minero de cobre en Argentina}

En base a un relevamiento realizado según las regiones argentinas se sabe que la región de Cuyo sin lugar a dudas tiene las mayores expectativas en recursos minerales. Algunos se encuentran en la etapa de estudios de factibilidad y otros en la faz exploratoria, siendo muy favorables las posibilidades de que algunos pasen a la etapa explotación. La información disponible se detalla por regiones o provincias [7-8]:

- La Rioja: en esta provincia existen depósitos metalíferos, cuya mineralización es de características vetiformes de $\mathrm{Au}-\mathrm{Cu}$. Incluye las minas La Mejicana, Los Bayos y Offir. En cambio, en el sector La Estrechura hay mena diseminada de Cu-Mo asociada a un stock dacítico terciario. Si bien no existen cálculos de reservas, en La Estrechura se estimaron 300.000 .000 t, con leyes de $0,06 \%$ Mo y $0,5 \% \mathrm{Cu}$, mientras que en La Mejicana se estimaron $250.000 \mathrm{t}$ con ley de 8,4 g/t Au.

- Mendoza: entre los yacimientos se destacan: Yalguaraz que es un prospecto epi a mesotermal de $\mathrm{Cu}$ y $\mathrm{Au}$. $\mathrm{Si}$ bien no se conocen las reservas, los recursos son de carácter promisorio. Paramillos Norte es un yacimiento de $\mathrm{Cu}-\mathrm{Au}-\mathrm{Mo}$. En este caso no se definieron aún los recursos, pero se estima que el sistema vetiforme contiene las mayores expectativas con más de $2.000 .000 \mathrm{t}$ y un sector de brechas con craquelación superaría las 50.000.000 t. En Paramillos Sur existe un pórfido cuprífero de $\mathrm{Cu}-\mathrm{Mo}-\mathrm{Au}$. Se han estimado reservas del orden de los $150.000 .000 \mathrm{t}$ con $0,58 \%$ $\mathrm{Cu}$ y 0,06-0,4 g/t Au. El yacimiento San Jorge es un pórfido cuprífero en el que se definieron reservas por 146.000.000 t con $0,5 \%$ Cu y $0,2 \mathrm{~g} / \mathrm{t} \mathrm{Au}$, y en la zona primaria se estimaron $30.000 .000 \mathrm{t}$ con $0,8 \% \mathrm{Cu}$. Cabe mencionar que dicho yacimiento se encuentra en exploración. 
- San Juan: el potencial minero metalífero reside en diversos tipos de yacimientos. El área del Carmen tiene mineralización diseminada de $\mathrm{Au}-\mathrm{Cu}$ en transición entre epitermal de alta sulfuración y depósito de tipo faja de Maricunga. Pachón es un pórfido cuprífero. La mineralización de $\mathrm{Cu}$-Mo está en vetas, diseminación y de tipo yacimiento fisural o "stockwork". Se consideran 687.000 .000 t con $0,63 \%$ Cu y 0,014\% Mo. Cuenta con un estudio de factibilidad.

- San Luis: el potencial minero metalífero está dado por variados yacimientos entre los cuales tenemos: Las Aguilas, que es un yacimiento de $\mathrm{Ni}-\mathrm{Co}-\mathrm{Cu}$ de tipo sulfuro masivo. Los estudios metalúrgicos demostraron la viabilidad técnica del procesamiento. Se delimitaron reservas totales de $2.220 .000 \mathrm{t}$ (probadas + probables). La ley media es $0,51 \% \mathrm{Ni}, 0,5 \% \mathrm{Cu}$ y 0,035 Co.

Como se puede observar según los datos del relevamiento mencionado anteriormente los yacimientos cupríferos en esta zona son de diversa índole y leyes. Otra zona rica en minerales cupríferos es la del Noroeste Argentino. Se destaca la puesta en marcha del primer yacimiento tipo pórfido cuprífero (Bajo de La Alumbrera de $\mathrm{Cu}-\mathrm{Au}$ ) [7, 9].

- Catamarca: se destacan las mineralizaciones metalíferas como El Bajo de La Alumbrera, que es un pórfido de $\mathrm{Cu}$ y $\mathrm{Au}$ emplazado en el Complejo Volcánico Farallón Negro. Las reservas posibles del depósito son $752 \mathrm{Mt}$ con leyes de $0,51 \% \mathrm{Cu}$ y 0,65 $\mathrm{g} / \mathrm{t} \mathrm{Au}$, con recuperación como subproductos de $\mathrm{Ag} \mathrm{y}$ Mo. La producción de concentrados, con un promedio de 180.000 t de Cu y 640.000 oz de Au/año. Dentro del mencionado complejo existen además varios prospectos de pórfidos cupríferos: Bajo del Durazno, Bajo de San Lucas, Bajo de los Jejenes, Bajo de las Pampitas, Agua Tapada, Bajo del Espanto. Uno de los más sobresalientes entre los depósitos en exploración es Agua Rica, que presenta una mineralización de $\mathrm{Au}, \mathrm{Cu}$ y Mo de tipo pórfido cuprífero y otra de tipo epitermal, con reservas de $802 \mathrm{Mt}$ y leyes de $0,61 \%$ de $\mathrm{Cu}, 0,035 \%$ de Mo y 0,24 g/t de Au. En Cerro Atajo la mineralización epitermal polimetálica está representada por $\mathrm{Cu}, \mathrm{Au}, \mathrm{Bi}, \mathrm{Sn}$ y $\mathrm{Ag}$.

Finalmente, analizando la región patagónica, las distintas unidades morfoestructurales presentes permiten una diversidad de recursos que van desde los energéticos a los metalíferos y no metalíferos en general [7].
- Neuquén: posee depósitos como Campana Mahuida que es un pórfido cuprífero con $\mathrm{Cu}-\mathrm{Au}-\mathrm{Mo}$. Se han evaluado $4.700 .000 \mathrm{t}$ con $0,73 \% \mathrm{Cu}$ en la zona oxidada y 33.000.000 t con $0,61 \% \mathrm{Cu}$ en la zona supergénica, con ley uniforme de 2,3 g/t Au y 132 ppm Mo. El depósito La Voluntad es un pórfido cuprífero, en el que hay mineralización diseminada y en un yacimiento fisural o "stockwork" de Cu-Mo. Se desconocen las reservas, sólo existen valores de leyes indicativas de $0,2 \% \mathrm{Cu}$.

- Santa Cruz: el potencial minero metalífero está representado por Arroyo Correntoso consistente en una veta mesotermal de $\mathrm{Pb}-\mathrm{Zn}-\mathrm{Ag}-\mathrm{Cu}-\mathrm{Au}$, a la que acompaña también una mineralización diseminada. Se han estimado reservas por 3.000.000 t con leyes promedio de $1,8 \% \mathrm{Zn}, 0,5 \% \mathrm{~Pb}, 0,5 \% \mathrm{Cu}, 0,6 \mathrm{~g} / \mathrm{t} \mathrm{Au}$ y $5,5 \mathrm{~g} / \mathrm{t} \mathrm{Ag}$.

- Chubut: el potencial minero metalífero está representado principalmente por depósitos polimetálicos. Mina Angela incluye las vetas de la mina Angela, de Clara Natividad y de Susana Beatriz. Se trata de depósitos polimetálicos vetiformes ( $\mathrm{Pb}-\mathrm{Zn}-\mathrm{Cu}-\mathrm{Au}-\mathrm{Ag})$. Se calcularon reservas de $350.000 \mathrm{t}$ de mineral probado con leyes de 5,6\% Zn, 2,9\% Pb, 0,38\% Cu, $50 \mathrm{~g} / \mathrm{t} \mathrm{Ag}$ y 3,5 g/t Au. El depósito Lago Fontana consiste en vetas polimetálicas mesotermales de $\mathrm{Zn}-\mathrm{Pb}-\mathrm{Ag}-\mathrm{Cu}$. Se determinaron reservas de 150.000 t con leyes de $4,5 \% \mathrm{Zn}, 1,6 \% \mathrm{~Pb}, 0,6 \% \mathrm{Cu}, 14 \mathrm{~g} / \mathrm{t} \mathrm{Ag}$ y $1 \mathrm{~g} / \mathrm{t} \mathrm{Au}$.

- Tierra del Fuego: la mina Beatriz es un depósito de sulfuros masivos polimetálicos ( $\mathrm{Zn}-\mathrm{Pb}-\mathrm{Cu}-\mathrm{Au}-\mathrm{Ag}) . \mathrm{Si}$ bien se desconocen datos de las reservas, una muestra de la galería de la mina analizada arrojó valores de $0,9 \% \mathrm{~Pb}, 3,4 \% \mathrm{Zn}$ y $40 \mathrm{~g} / \mathrm{t} \mathrm{Ag}$. En la provincia existen otras áreas, a la fecha en exploración, denominadas Arroyo Rojo, Sargent, Río Hambre, Río Encajonado, Gregores y Lago Guanaco. En muestras de superficie con mineralización estratiforme masiva se obtuvieron leyes medias de $8,62 \% \mathrm{Zn}, 1,79 \% \mathrm{~Pb}, 0,89 \% \mathrm{Cu}, 0,4$ g/t Au y 37,2 g/t Ag.

Como se puede ver, la producción de cobre a obtener en toda la República Argentina tiene una ley de aproximadamente $0,65 \%$. Si se compara con leyes informadas en países vecinos tales como Chile y Brasil aproximadas al 0,5\%, se puede pensar en que Argentina podría participar junto a ellos del mercado del cobre en forma competitiva. 


\section{ESTUDIO DE CONCENTRADOS DE MINERALES DE COBRE}

De acuerdo a lo mencionado previamente, Argentina posee un gran potencial minero, el cual se halla en este momento en diferentes etapas de exploración y explotación. Actualmente existen yacimientos en el norte de nuestro país que producen concentrados de cobre desde minerales como calcopirita y calcosina-covelina [1]. Por lo que en este trabajo se estudian muestras de baja ley de cobre que ingresan al proceso de concentración en Catamarca, que constituye el yacimiento de cobre en operación de mayor envergadura en Argentina.

\section{Estudio microestructural}

El estudio microestructural se realizó sobre muestras de mineral que ingresa actualmente a la mina y posteriormente es tratado mediante flotación "rougher" para obtener los concentrados de cobre. Resulta relevante en este estudio poder determinar los distintos constituyentes minerales en los concentrados e identificar las fases presentes en las partículas. Esta información es necesaria para el diseño posterior de los procesos industriales. A tal fin, se observa la estructura de dichas partículas mediante microscopía óptica. En las Figuras 2 y 3 se presentan detalles de las imágenes obtenidas.

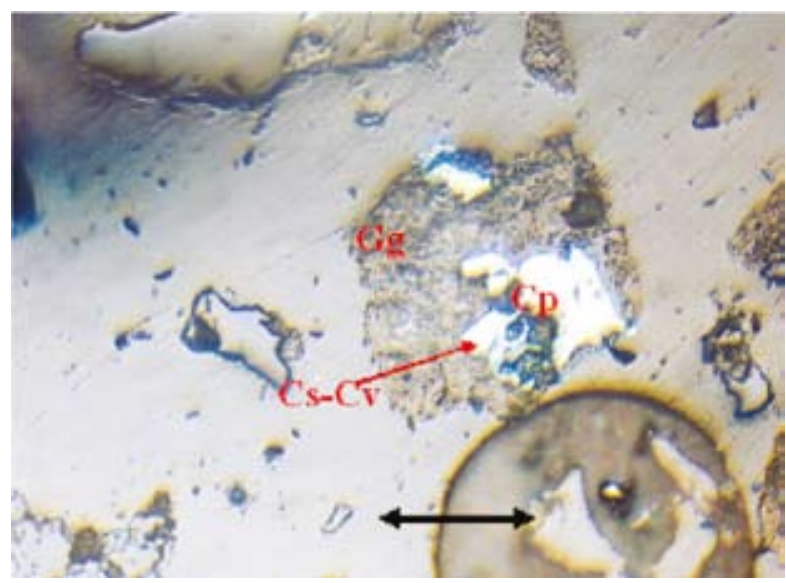

Figura 2. Partículas de ganga (Gg) ligadas a calcopirita (Cp) y calcosina-covelina (Cs-Cv) de color azul (100\#) observadas en la muestra de concentrado en estudio [M16x]. Escala $100 \mu \mathrm{m}$.

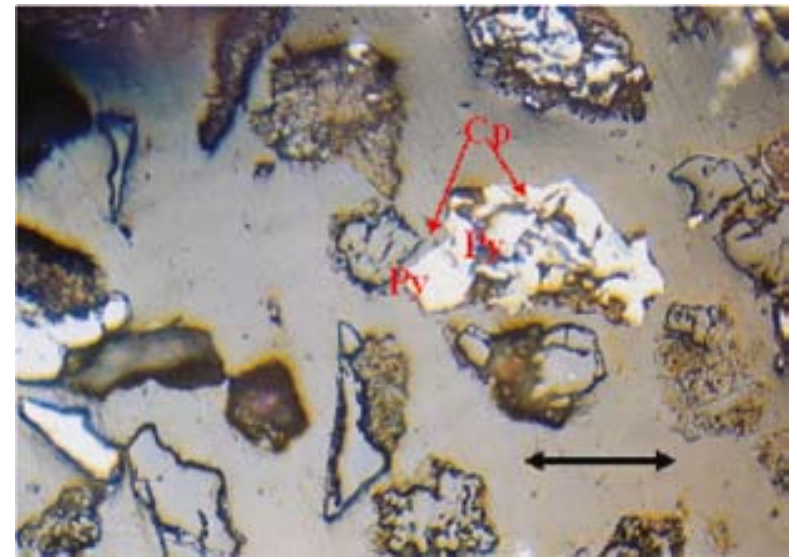

Figura 3. Partícula de calcopirita $(\mathrm{Cp})$ asociada a pirita (Py) y a calcosina-covelina (Cs-Cv) de color azul (-150+200\#) [M16x]. Escala $100 \mu \mathrm{m}$.

Tal como se puede apreciar, se ha comprobado que las partículas presentes en el mineral son de tipo calcopirita (Figura 2), se identifican partículas de ganga (Gg) asociadas a calcopirita y partículas de calcosina-covelina que se visualizan de color azul. En la Figura 3 se observa un mineral de tipo calcosina-covelina con partículas de calcopirita (Cp), que se hallan asociadas a pirita (Py) y calcosina-covelina (Cs y $\mathrm{Cv})$ respectivamente.

\section{Composición química}

En la Tabla 1 se presentan las composiciones químicas medias de la producción de los concentrados obtenidas desde el año 2001 hasta el 2008 en nuestro país.

Tabla 1. Composición medias de producción de concentrados.

\begin{tabular}{|l|l|l|l|l|l|l|l|l|}
\hline \multicolumn{1}{c|}{} & $\mathbf{2 0 0 1}$ & $\mathbf{2 0 0 2}$ & $\mathbf{2 0 0 3}$ & $\mathbf{2 0 0 4}$ & $\mathbf{2 0 0 5}$ & $\mathbf{2 0 0 6}$ & $\mathbf{2 0 0 7}$ & $\mathbf{2 0 0 8}$ \\
\hline $\mathrm{Cu}$ & 27,8 & 27,4 & 26,5 & 27,0 & 27,0 & 26,8 & 25,6 & 24,0 \\
\hline $\mathrm{Fe}$ & 28,3 & 29,4 & 29,7 & 29,7 & 29,5 & 28,8 & 28,3 & 30,1 \\
\hline $\mathrm{S}$ & 33,3 & 33,5 & 34,0 & 33,7 & 33,8 & 33,8 & 34,5 & 35,3 \\
\hline $\mathrm{S} / \mathrm{Cu}$ & 1,20 & 1,22 & 1,28 & 1,25 & 1,25 & 1,26 & 1,35 & 1,47 \\
\hline
\end{tabular}

En la Tabla 2 se puede observar la composición química típica usada por Moraga [10], Moskalk [11], Sanchez [12] y Dimitrijevic [13] de los concentrados usados en las fundiciones de cobre de otros países. 
Tabla 2. Composición de concentrados usados en las fundiciones.

\begin{tabular}{|l|c|c|c|c|c|c|c|c|c|}
\hline \multicolumn{1}{|c|}{$\%$} & $\mathbf{T}-1$ & $\mathbf{T}-2$ & $\mathbf{T}-3$ & $\mathbf{T}-4$ & $\mathbf{T}-5$ & $\mathbf{T}-6$ & $\mathbf{T}-7$ & $\mathbf{T}-8$ & $\mathbf{T}-9$ \\
\hline $\mathrm{Cu}$ & 34,09 & 33,10 & 32,11 & 31,11 & 30,12 & 29,13 & 28,14 & 27,15 & 26,16 \\
\hline $\mathrm{S}$ & 30,68 & 31,45 & 32,11 & 32,67 & 33,13 & 33,50 & 33,77 & 33,94 & 34,01 \\
\hline $\mathrm{Fe}$ & 24,80 & 25,03 & 25,35 & 25,79 & 26,31 & 26,94 & 27,66 & 28,48 & 29,40 \\
\hline $\mathrm{SiO}_{2}$ & 5,30 & 5,30 & 5,30 & 5,30 & 5,30 & 5,30 & 5,30 & 5,30 & 5,30 \\
\hline $\mathrm{Al}_{2} \mathrm{O}_{3}$ & 3,40 & 3,40 & 3,40 & 3,40 & 3,40 & 3,40 & 3,40 & 3,40 & 3,40 \\
\hline $\mathrm{CaO}^{2}$ & 0,90 & 0,90 & 0,90 & 0,90 & 0,90 & 0,90 & 0,90 & 0,90 & 0,90 \\
\hline $\mathrm{MgO}$ & 0,50 & 0,50 & 0,50 & 0,50 & 0,50 & 0,50 & 0,50 & 0,50 & 0,50 \\
\hline $\mathrm{Otros}$ & 0,33 & 0,33 & 0,33 & 0,33 & 0,33 & 0,33 & 0,33 & 0,33 & 0,33 \\
\hline $\mathrm{S} / \mathrm{Cu}$ & 0,90 & 0,95 & 1,00 & 1,05 & 1,10 & 1,15 & 1,20 & 1,25 & 1,30 \\
\hline
\end{tabular}

\section{ANÁLISIS DE RESULTADOS}

Según Davenport [14], Sancho [15] y Vanini [16] confirman que la calcopirita es uno de los minerales de $\mathrm{Cu}$ más extensamente distribuidos en la naturaleza, conocido también como pirita de $\mathrm{Cu}$ y cuya fórmula es $\mathrm{FeCuS}_{2}$. $\mathrm{La}$ covelina $(\mathrm{CuS})$ y la calcosina $\left(\mathrm{Cu}_{2} \mathrm{~S}\right)$ constituyen dos tipos de sulfuros de cobre también abundantes en la naturaleza.

Resulta evidente a partir de los resultados mencionados en la Tabla 1 que las calidades de concentrados de cobre varían a lo largo de los años de producción. En particular, en los concentrados contemplados en este estudio se observa una disminución del porcentaje de $\mathrm{Cu}$ y en consecuencia un incremento progresivo de la relación $\mathrm{S} / \mathrm{Cu}$ (Figuras 4 y 5).

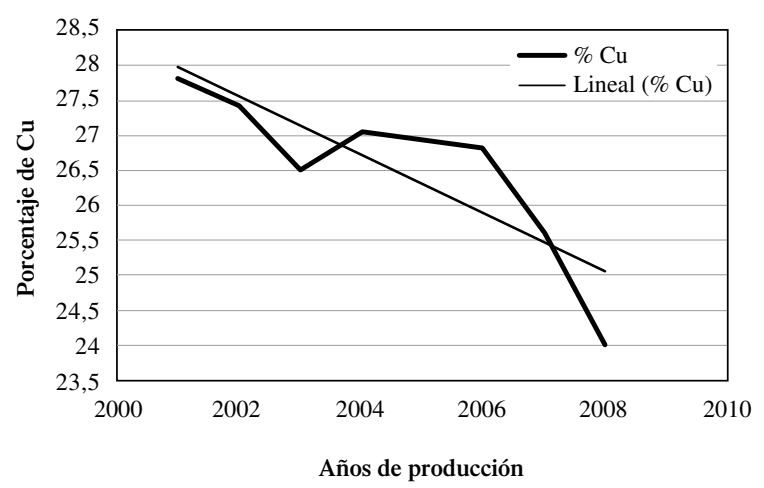

Figura 4. Evolución del porcentaje de $\mathrm{Cu}$ en los concentrados en estudio a lo largo de los años.

Existen actualmente proyectos mineros que pueden aportar aumentos en la media de estos parámetros en la producción argentina.
Realizando un análisis en función de la composición química de los concentrados de cobre utilizados habitualmente en las fundiciones de cobre se puede pensar que los concentrados obtenidos a través de la producción nacional se encuadran en la clasificación T7-T9.

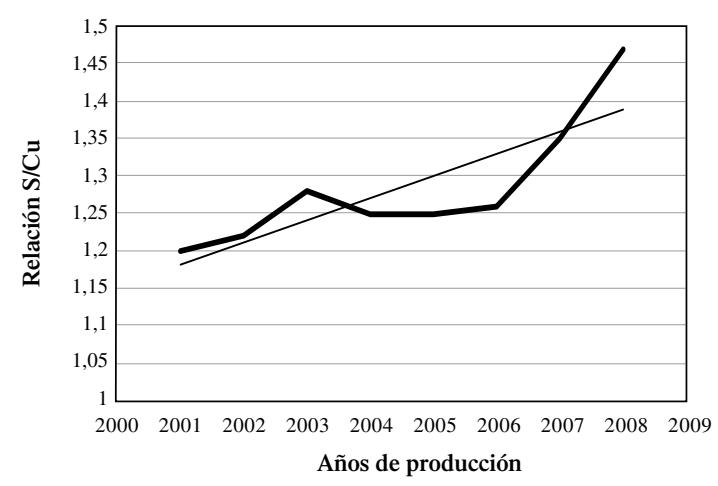

Figura 5. Evolución del porcentaje de $\mathrm{Cu}$ en los concentrados en estudio a lo largo de los años.

Según los datos proporcionados por Moraga (2007), con la relación de $\mathrm{S} / \mathrm{Cu}$ que varía desde 1,20 a 1,30, se pueden obtener ejes de $58 \%$ de cobre significando esto una capacidad máxima de producción de aproximadamente 70 tph. Sin embargo, si se quiere aumentar la calidad de eje resulta necesario bajar nuestra tasa de fusión, como se observa en la Figura 6. El producto final de las fundiciones de cobre es el ánodo, material con contenidos de cobre del orden de 99,4 a 99,8\%. Esta composición química aún no permite alcanzar las propiedades eléctricas y mecánicas que se requieren y que se logran con un cobre de mayor pureza. Por tal razón, el cobre anódico necesariamente debe pasar por un proceso de refinación adicional, proceso llamado electrorrefinación; el producto obtenido es de 99,99\% de $\mathrm{Cu}$.

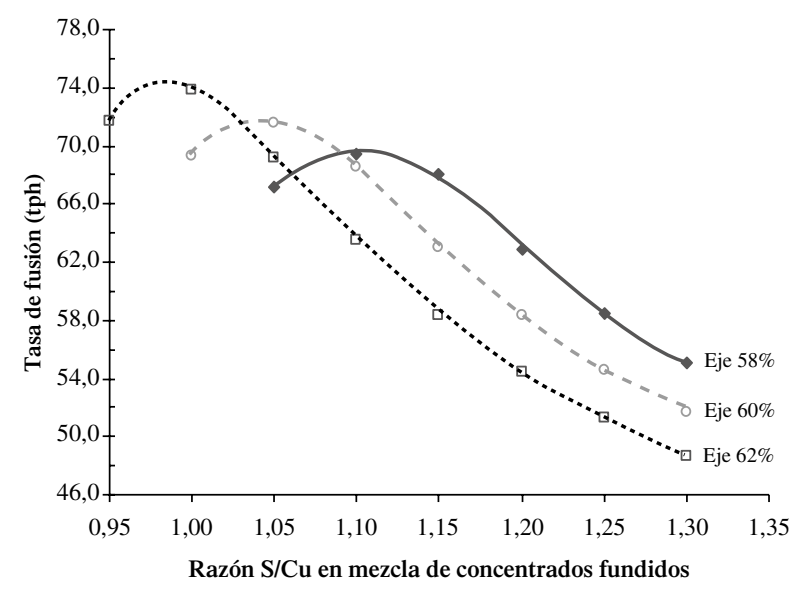

Figura 6. Tasa de fusión en función de la relación $\mathrm{S} / \mathrm{Cu}$. 
Normalmente se considera que a mayor producción, entendida como la cantidad de cobre procesado por la fundición, mayores serán sus utilidades. Este concepto es erróneo ya que los ingresos de una fundición quedan básicamente definidos por el diferencial que existe entre el tratamiento de la carca y la carga del proceso de refino denominada TCRC (treatment charge-refining charge) del concentrado menos el RC del producto (refining charge). Por lo tanto, los ingresos de la fundición se verán incrementados en la medida que aumente la cantidad de concentrado fundido, ya que de este modo la fundición recibirá un mayor ingreso por la aplicación de cargos de tratamiento a los concentrados. Por el contrario, el incremento del contenido de cobre fundido, y por ende producido como ánodo o blíster, efectivamente aumenta el monto del cargo de refinación al concentrado. Esto significa que también aumenta en igual proporción el cargo de refinación del ánodo o blíster que aplicarán las refinerías electrolíticas.

\section{CONCLUSIONES}

A partir de los resultados de este estudio se puede inferir que:

Si bien Argentina hasta el momento ha realizado una explotación parcial de su potencial cuprífero, obteniendo concentrados de leyes atractivas para los mercados extranjeros, podría a través del desarrollo de la industria de fundición de cobre alcanzar una trayectoria similar a la lograda por los países vecinos.

El relevamiento sobre el potencial minero es concluyente en cuanto al gran potencial minero existente, de manera que para aprovecharlo plenamente se considera vital comenzar a desarrollar acciones más intensivas orientadas a la promoción y desarrollo de nuevos mercados en el marco de las contempladas por el International Copper Association, que podría significar duplicar el presupuesto en un período de cinco años.

Los estudios realizados sobre muestras minerales han permitido comprobar que los mismos resultan de buena calidad y bajo porcentaje de impurezas tales como As, $\mathrm{Sb}$ y $\mathrm{Bi}$, que son consideradas muy nocivas para la producción de cobre refinado debido a la emanación de gases perjudiciales para el medio ambiente.

En base a los resultados de los estudios realizados sobre los concentrados se puede pensar que los mismos proporcionarían una buena rentabilidad en cuanto a la producción de cobre. El próximo paso a considerar a partir de estos resultados sería realizar un estudio de costos actualizado de una planta de fundición de cobre en nuestro país y evaluar el impacto directo de la misma sobre la economía y el medio social de la región que conlleva a la formación de recursos humanos, la promoción de nuevos proyectos industriales y de servicios, dando una importante contribución para el crecimiento y la inserción de Argentina en el mercado del cobre a nivel mundial.

\section{AGRADECIMIENTOS}

Se agradece el apoyo del Consejo Nacional de Investigaciones Científicas y Técnicas, Argentina (CONICET), a la labor de investigación.

\section{REFERENCIAS}

[1] A. Gutiérrez, G. Chong y S. Espinoza. "Niveles de exposición de yacimientos del distrito minero de Agua de Dionisio (YMAD), Catamarca". Revista de la Asociación Geológica Argentina. Vol. 61 No 2, pp. 269-277. 2006.

[2] E. Peralta. "Actas de Encuentro Internacional de Minería". Secretaría de Minería de la Nación. Buenos Aires. Argentina, pp. 5-15.1994.

[3] G. Preisz. "El Norte de la Minería Argentina". Ediciones Panorama Minero, pp. 162-174. 2000.

[4] J.F. Papp, E. Lee Bray, D. L. Edelstein, M.D. Fenton, D.E. Guberman, J.B. Hedrick, J.D. Jorgenson, P.H. Kuck, K.B. Shedd and A.C. Tolcin. "Factors that influence the price of $\mathrm{Al}, \mathrm{Cd}, \mathrm{Co}, \mathrm{Cu}, \mathrm{Fe}, \mathrm{Ni}, \mathrm{Pb}$, Rare Earth Elements, and Zn”. U.S. Geological Survey Open-File Report 2008-1356. 2008.

[5] Scavage. "Exportación e importación de cobre en Argentina". Fecha de consulta: 22 de agosto del 2009. URL: http://www.scavage.com/trade? menu=ar.import\&query=product:cobre

[6] A. Beretta y V. Bazán. "Algunos fundamentos de las ventajas estratégicas de la instalación de una fundición de cobre en Argentina" SAM/ CONAMET, pp. 7-12. San Nicolás. Buenos Aires, Argentina, 2007.

[7] Servicio Geológico Minero Argentino. “Oferta Minera. Regiones Mineras. Datos”. Fecha de consulta: 10 de agosto de 2009. URL: http://www. segemar.gov.ar/P_Oferta_Regiones/Regiones/ index.htm

[8] San Juan Gobierno-Minería. "Recursos mineros. Metales preciosos y de base". Fecha de consulta: 12 de agosto de 2009. URL: http://mineria.sanjuan. gov.ar/recursos/proyectos/ProyectoPachon.pdf

[9] Catamarca Gobierno-Minería. "Proyectos en marcha. Minera Alumbrera". Fecha de consulta: 15 de agosto 
de 2009. URL: http://www.mineriacatamarca.gov. ar/indexmozilla.html

[10] R. Moraga. "Modelo Técnico Económico para la Creación de valor en las Fundiciones de Concentrado de Cobre". Tesis para optar al grado de Ingeniero. Universidad de Concepción. Concepción, Chile. pp. 50-67. 2007.

[11] R. Moskalk and A. Alfantazi. "Review of copper pyrometallurgical practice: today and tomorrow". Mineral Engineering. Vol. 16, Issue 10, pp. 893919. October, 2003.

[12] V. Sanchez, J. Valero, P. Flores and M. Perez. "Mass Balance Calculation in Copper Flash Smelting by Means of Genetic Algorithms". JOM. Vol. 56, Issue 12, pp. 29-32. December, 2004.
[13] M. Dimitrijevic, A. Rostov, V. Tasic and N. Melosevic. "Influence of pyrometallurgical copper production on the environment". Journal of Hazardous Materials. Vol. 164, Issues 2-3, pp. 892-899. May 30, 2009.

[14] W.G. Davenport, M. King, M. Schlesinger and A. K. Biswas. "Extractive metallurgy of copper". Four edition. Pergamon Press, pp. 97-114. 2000.

[15] J.P. Sancho, L.F. Verdeja y A. Ballester. "Metalurgia Extractiva”. Ed. Síntesis, Vol. II. pp. 245-294. Madrid, España. 2000.

[16] G. Vanini. "Introducción a la metalurgia. Recursos metalúrgicos: metalurgia general II". E.A.P. de Ingeniería Metalúrgica Universidad Nacional Mayor de San Marcos, pp. 146-170. Lima, Perú. 2004. 